\title{
SIMPLE-CONNECTIVITY AND THE BROWDER-NOVIKOV THEOREM $\left(^{1}\right)$
}

\author{
BY \\ M. KERVAIRE AND A. VASQUEZ
}

In this note we construct a family of odd-dimensional, closed, combinatorial manifolds, none of which has the homotopy type of a closed differentiable manifold. These manifolds all have an infinite cyclic fundamental group.

W. Browder [1] and S. P. Novikov [9] have proved that a simply-connected finite complex, $K$, satisfying Poincaré duality with respect to an odd-dimensional fundamental class $u \in H_{2 n+1}(K),(n \geqq 2)$ has the homotopy type of a closed $(2 n+1)$ dimensional differential manifold provided there exists a vector bundle, $\xi$, over $K$ whose Thom space $T(\xi)$ has a spherical top homology class (i.e., $\pi_{q} T(\xi) \rightarrow H_{q} T(\xi)$ is surjective for $q=2 n+1+\operatorname{dim} \xi$ ).

Denoting one of our combinatorial manifolds by $M$, we prove that SM, the suspension of $M$, has a spherical top homology class, so that the trivial real line bundle over $M$ satisfies the Browder-Novikov hypothesis. The manifolds, $M$, show that the Browder-Novikov theorem cannot be extended to the nonsimplyconnected case, at least not without additional hypotheses on $K$. (Recall that the Thom space of the trivial real line bundle over a space $X$ has the homotopy type of $S^{1} \vee S X$.)

The manifolds $M^{2 n+1}$ are constructed from certain knotted homotopy (2n-1)spheres in $S^{2 n+1}$ ( $n$ is $o d d$ ). Let $A$ be (the space of) the tangent unit disk bundle over $S^{n}$, and $W$ the differential manifold with boundary obtained by plumbing together two copies $A_{1}, A_{2}$ of $A$. (See [4], [5], or [8].) The images $S_{1}, S_{2}$ in $W$ of the zero cross-sections in $A_{1}$ and $A_{2}$ respectively have a single (transversal) intersection point. Denote by $\Sigma^{2 n-1}$ the boundary of $W$. It was proved in [8], or more generally follows from Smale theory, that $\Sigma^{2 n-1}$ is combinatorially equivalent to $S^{2 n-1}$.

We now imbed $W$ into $S^{2 n+1}$. It is well known, and easy to see, that $W$ can be differentiably imbedded into $S^{2 n+1}$ so that if $\nu$ denotes a normal vector-field on $W$ in $S^{2 n+1}$, and if $S_{1}^{\prime}, S_{2}^{\prime}$ are the translates of $S_{1}, S_{2}$ by a small positive amount $\varepsilon$ along $\nu$, then $\lambda_{i}=L\left(S_{i}, S_{i}^{\prime}\right)$ for $i=1,2$, are odd integers, where $L($, ) denotes the linking coefficient in $S^{2 n+1}$. (For further remarks on this see the lemma on normal bundles at the end of this paper.)

Received by the editors August 16, 1966.

(1) This paper represents results obtained under the sponsorship of the Office of Naval Research, Contract NONR-285 (46) at the Courant Institute of Mathematical Sciences, New York University, and the National Science Foundation, Contract NSF-GP 4598 at Brandeis University. 
Let $\phi: \Sigma^{2 n-1} \times D^{2} \rightarrow S^{2 n+1}$ be a tubular neighborhood of $b W$, the boundary of $W$, and let $h: S^{2 n-1} \rightarrow \Sigma^{2 n-1}$ be a combinatorial equivalence. Then

$$
\psi: S^{2 n-1} \times D^{2} \rightarrow S^{2 n+1}
$$

given by $\phi \circ(h \times$ id $)$ is a combinatorial imbedding. Let $M^{2 n+1}=\chi\left(S^{2 n+1}, \psi\right)$ be the combinatorial manifold obtained from $S^{2 n+1}$ by spherical modification:

$$
M^{2 n+1}=\left(S^{2 n+1}-\psi\left(S^{2 n-1} \times B^{2}\right)\right) \cup D^{2 n} \times S^{1},
$$

where $B^{2}=$ int $D^{2}$, and $(x, y) \in S^{2 n-1} \times S^{1} \subset D^{2 n} \times S^{1}$ is identified with $\psi(x, y)$. As usual we denote by $\psi^{\prime}: D^{2 n} \times S^{1} \rightarrow M$ the imbedding induced by the inclusion.

PROPOSITION. The manifolds $M^{2 n+1}$ constructed above for odd $n$ have the following properties for $n>1$ :

(1) $\pi_{i} M \cong \pi_{i}\left(S^{1}\right)$ for $i<n$;

(2) $\pi_{n} M$ as a $Z[J]$-module, where $J=\pi_{1}\left(S^{1}\right)$ generated by $t$, is isomorphic to the $Z[J]$-module with presentation

$$
\left\{x_{1}, x_{2} ; \lambda_{1}(t-1) x_{1}+(\lambda(t-1)+1) x_{2},(\lambda(t-1)-t) x_{1}+\lambda_{2}(t-1) x_{2}\right\} .
$$

(Here $\lambda$ is an integer depending on the imbedding $W \rightarrow S^{2 n+1}$, and can be chosen arbitrarily.)

(3) $H_{*}(M) \cong H_{*}\left(S^{1} \times S^{2 n}\right)$;

(4) $\pi_{2 n+2}(\mathrm{SM}) \rightarrow H_{2 n+2}(\mathrm{SM})$ is surjective, where $\mathrm{SM}$ denotes the suspension of $M$.

(5) For $n=3,7$ the combinatorial equivalence $h: \Sigma^{2 n-1} \rightarrow S^{2 n-1}$ can be taken to be a diffeomorphism so that the manifold $M^{2 n+1}$ has a differential structure. For $n=4 k+1, M^{2 n+1}$ does not have the homotopy type of any closed differential manifold.

REMARK. Since isomorphic $Z[J]$-modules have identical elementary ideals, and the 0 th elementary ideal of the module under (2), i.e., the ideal generated by the determinant of the relation matrix, is $\left(\lambda_{1} \lambda_{2}-\lambda^{2}+\lambda\right)(t-1)^{2}+t$ it follows that by varying the coefficients $\lambda_{1}, \lambda_{2}, \lambda$ we can get infinitely many distinct homotopy types for the manifold $M^{2 n+1}$.

The proofs of (1) and (2) rely on the method for calculating the homotopy groups of the complement of a knot, exposed in [4]. It is clear that

$$
M-\psi^{\prime}\left(D^{2 n} \times S^{1}\right)=S^{2 n+1}-\psi\left(S^{2 n-1} \times D^{2}\right) .
$$

Hence $\pi_{i} M=\pi_{i}\left(S^{2 n+1}-b W\right)$ for $i<2 n-1$. Now, a homomorphism

$$
I: \pi_{1}\left(S^{2 n+1}-b W\right) \rightarrow Z
$$

is given by assigning to $\alpha \in \pi_{1}\left(S^{2 n+1}-b W\right)$ the intersection coefficient with $W$ of a representative curve $f: S^{1} \rightarrow S^{2 n+1}-b W$. Clearly, $I$ is surjective. To prove that $I$ is injective, let $U$ be a tubular neighborhood of $W$ and let $Y=S^{2 n+1}-U$. Then first, $\pi_{1} Y=\{1\}$ by the van Kampen theorem, since $W$ and hence $b Y$ are simply connected. Secondly, $\pi_{1}(Y, b Y)=\{1\}$ by the homotopy exact sequence of $(Y, b Y)$. 
An element $\alpha \in \operatorname{Ker} I$ can be represented by a differentiably imbedded curve $[0,1] \rightarrow S^{2 n+1}-b W$ which intersects $W$ transversally in a finite number of interior points. Since $I(\alpha)=0$, there will be a pair of consecutive intersection points with $W$ having oppositive intersection coefficients. The arc joining these points represents an element of $\pi_{1}(Y, b Y)$ and thus is homotopic to an arc in $U$ which can then be pushed away from $W$, reducing the number of intersection points by 2 . Eventually, we get a representative of the given element $\alpha$ whose image is contained in $Y$. Since $Y$ is simply connected, $\alpha=1$.

To calculate $\pi_{i} M$ for $i \leqq n$, denote by $W_{+}$and $W_{-}$the two copies of $W$ in $b U=b Y$. Let $X_{1}=S^{2 n+1}-b W$. Following Seifert, we construct the universal covering $X$ of $X_{1}$ as the union of countably many copies of $Y$ which we denote by $Y^{k}, k \in Z$, with $W_{+}^{k}$ identified with $W_{-}^{k+1}$ for every $k$. Then $\pi_{i}\left(S^{2 n+1}-b W\right)=0$ for $2 \leqq i<n$ follows immediately from $H_{i} Y=0$ for $i<n$. The module $\pi_{n}\left(S^{2 n+1}-b W\right)$ is isomorphic to $H_{n}(X)$ for which we get a presentation by the Mayer-Vietoris theorem. If we denote by $\xi_{1}, \xi_{2}$ the generators of $H_{n} W$ represented by $S_{1}, S_{2}$ respectively, $\nu_{+}$and $\nu_{-}$the obvious mappings of $W$ onto $W_{+}$and $W_{-}$, then a presentation for $H_{n}(X)$ is obtained from a presentation of $H_{n} Y$ (as an abelian group) by adjoining the relations $t \nu_{+}\left(\xi_{i}\right)=\nu_{-}\left(\xi_{i}\right)$. Now, it is easy to see that $H_{n} Y$ is free abelian on 2 generators $x_{1}, x_{2}$, and if $S$ is a sphere in $Y$, the class of $S$ is given by

$$
(S)=L\left(S, S_{1}\right) x_{1}+L\left(S, S_{2}\right) x_{2}
$$

The presentation for $H_{n}(X) \cong \pi_{n} M$ claimed in (2) follows readily, with $\lambda=L\left(S_{1}^{\prime}, S_{2}\right)$.

The proof of (3) is immediate.

To prove (4) we first observe that $M$ has the homotopy type of a cell complex of the form

$$
K=\left(S^{1} \vee S_{1}^{n} \vee \cdots \vee S_{\alpha}^{n}\right) \cup e_{1}^{n+1} \cup \cdots \cup e_{\alpha}^{n+1} \cup e^{2 n} \cup e^{2 n+1} .
$$

(In fact, it can be proved using Smale theory that $M$ has a handle decomposition inducing the above cell structure. See [4].) Since $H_{n} M=0$, we have, up to homotopy type

$$
\mathrm{SM}=\left(S^{2} \cup_{f} e^{2 n+1}\right) \cup_{g} e^{2 n+2} .
$$

Now, since $H^{1} M=Z$, there is a map $M \rightarrow S^{1}$ whose composition with the inclusion $S^{1} \rightarrow M$ is homotopic to the identity $S^{1} \rightarrow S^{1}$. Taking the suspension of these maps we see that $S^{2}$ is a retract of $M$. It follows that the attaching map $f$ is trivial, and thus

$$
\mathrm{SM}=\left(S^{2} \vee S^{2 n+1}\right) \cup_{g} e^{2 n+2},
$$

up to homotopy type. Since $H_{2 n+2} M=Z$, it follows that $g$ must have degree 0 (on $S^{2 n+1}$ ), and since $\pi_{2 n+1}\left(S^{2} \vee S^{2 n+1}\right)=\pi_{2 n+1}\left(S^{2}\right)+\pi_{2 n+1}\left(S^{2 n+1}\right), g$ is homotopic to a mapping $h$ into $S^{2}$. Thus SM has the homotopy type of

$$
S^{2 n+1} \vee\left(S^{2} \cup_{g h} e^{2 n+2}\right)
$$


Using again the retraction $\mathrm{SM} \rightarrow S^{2}$, we see that $h$ is trivial. So SM has the homotopy type of

(4) is now obvious.

$$
S^{2} \vee S^{2 n+1} \vee S^{2 n+2}
$$

The proof of (5) will rely essentially on recent results of E. Brown and F. Peterson [2]. Suppose $M^{2 n+1}$ is a closed differential manifold ( $n$ odd, $\neq 3,7$ ) with the properties (1), (2), and (3). We construct a knot as follows. Let $\phi: S^{1} \times D^{2 n} \rightarrow M$ be a differentiable imbedding representing the generator $t \in \pi_{1} M$. Performing a spherical modification we obtain a manifold $\Sigma^{2 n+1}=\chi(M, \phi)$ which is easily seen to be a differential homotopy sphere. Replacing $M^{2 n+1}$ by the connected sum $(M \#(-\Sigma))$ if necessary, we may assume that $\Sigma^{2 n+1}$ is diffeomorphic to $S^{2 n+1}$. (This operation is not in fact really necessary for what follows.) Since

$$
S^{2 n+1}=\left(M-\phi\left(S^{1} \times B^{2 n}\right)\right) \cup D^{2} \times S^{2 n-1},
$$

we have an imbedding $f: S^{2 n-1} \rightarrow S^{2 n+1}$. (It is however essential that $S^{2 n-1}$ here be the sphere with the usual differential structure.) An argument similar to the one used in the proof of (1) and (2) shows that $\pi_{i}\left(S^{2 n+1}-f\left(S^{2 n-1}\right)\right)=\pi_{i}\left(S^{1}\right)$ for $i<n$, and $\pi_{n}\left(S^{2 n+1}-f\left(S^{2 n-1}\right)\right)$ is the $Z[J]$-module whose presentation is given in (3). It is well known that $f\left(S^{2 n-1}\right)$ is the boundary of an orientable submanifold $V$ of $S^{2 n+1}$. Moreover J. Levine has proved that the manifold $V$ can be taken to be $(n-1)$-connected as a consequence of $\pi_{i}\left(S^{2 n+1}-f\left(S^{2 n-1}\right)\right)=\pi_{i}\left(S^{1}\right)$ for $i<n$. It follows from Poincaré duality (for $V$ ) that we may find a basis, $\left\{\xi_{1}, \ldots, \xi_{s}, \eta_{1}, \ldots, \eta_{s}\right\}$, for $H_{n}(V)$ having the intersection numbers $\delta_{i j}=I\left(\xi_{i}, \eta_{j}\right), O=I\left(\xi_{i}, \xi_{j}\right)=I\left(\eta_{i}, \eta_{j}\right)$. Using a normal vector field along $V$ we may imbed $V \times[-\varepsilon,+\varepsilon]$ in $S^{2 n+1}$ in such a way that $V$ corresponds to $V \times\{0\}$. If $\alpha \in H_{n}(V)$ we denote by $\alpha^{ \pm}$the elements of $H_{n}\left(S^{2 n+1}-V\right)$ represented by the corresponding class in $V \times\{ \pm \varepsilon\} \subset S^{2 n+1}-V$. Since any $\alpha \in H_{n}(V)$ can be represented by an imbedded sphere and any two such can be put in general position it is clear that $L\left(\alpha^{+}, \beta\right)-L\left(\alpha^{-}, \beta\right)=L\left(\alpha^{+}-\alpha^{-}, \beta\right)$ $=$ the intersection number of the chain $[-\varepsilon, \varepsilon] \times \alpha$ with $\beta=I(\alpha, \beta)$. It now follows from the methods used to establish (3) that there is a presentation of $\pi_{n}\left(S^{2 n+1}-\partial V\right)$ with $2 s$ generators and the relation matrix

$$
R=\left\|\begin{array}{cc}
(t-1) A & (t-1) B+E \\
(t-1) B^{\prime}-t E & (t-1) C
\end{array}\right\|
$$

where $E$ is the $s \times s$ identity matrix, $B^{\prime}$ is the transpose of $B$ and $A=\left\|L\left(\xi_{i}^{+}, \xi_{j}\right)\right\|$, $B=\left\|L\left(\xi_{i}{ }^{+}, \eta_{j}\right)\right\|$ and $C=\left\|C_{i j}\right\|=\left\|L\left(\eta_{i}{ }^{+}, \eta_{j}\right)\right\|$. (We remark that the matrices $A$ and $C$ are symmetric (because $n$ is odd).) Using the lemma below we recognize $a_{i 1} \bmod 2$ as the obstruction to trivializing the normal bundle in $V$ to an imbedded sphere representing $\xi_{i}$. A similar relation holds between $c_{i i}$ and $\eta_{i}$ and so

$$
c(V)=\sum_{i=1}^{s} a_{i i} c_{i i} \bmod 2
$$

is the Arf invariant of the quadratic form of $V$ as defined in [6]. 
Now, det $R$ is the 0th elementary ideal of $\pi_{n}(M)=\pi_{n}\left(S_{2 n+1}-f\left(S^{2 n-1}\right)\right)$ and hence det $R$ and $\left(\lambda_{1} \lambda_{2}-\lambda^{2}+\lambda\right)(t-1)^{2}+t$ must generate the same ideal in $Z[J]$. An argument of Robertello's [10], sketched below for the convenience of the reader, shows that det $R=t^{s-1}\left(c(V)\left(t^{2}+1\right)+t\right)$ modulo the ideal generated by 2 and $(t-1)^{4}$. Since $\left(\lambda_{1} \lambda_{2}-\lambda^{2}+\lambda\right)(t-1)^{2}+t=\left(t^{2}+1\right)+t \bmod 2$, it follows that $C(V)=1 \bmod 2$ but since $\partial V$ is diffeomorphic to $S^{2 n-1}$ this contradicts the Brown-Peterson result [2] when $n=4 k+1, k \geqq 1$.

Robertello's argument in brief is this. Let $R=\left(x_{i j}\right)$-thus $x_{i j}$ is divisible by $t-1$ except if $1 \leqq i \leqq s$ and $j=i+s$ or $1 \leqq j \leqq s$ and $i=j+s$. Let $S_{\alpha, \beta}$ be the set of permutations $\left(i_{1}, \ldots, i_{2 s}\right)$ for which $i_{k} \neq s+k$ for exactly $\alpha$ values of $k \in[1, s]$ and $i_{s+k} \neq k$ for exactly $\beta$ values of $k \in[1, s]$. Thus

$$
\operatorname{det} R=\sum_{0 \leqq \alpha, \beta \leqq k} \sum_{S_{\alpha, \beta}} x_{1, i_{1}} x_{2, i_{2}} \cdots x_{2 s, i_{2} s} \text {. }
$$

Now the individual terms in $s_{\alpha \beta}$ for $\alpha, \beta \in[2, s]$ are divisible by $(t-1)^{4}$ so that we need only consider the first few $S_{\alpha, \beta}$ 's. $S_{0,0}$ contains only the permutation $(s+1, \ldots, 2 s, 1,2, \ldots, s-1, s)$ which gives rise to the term

$$
\prod_{i=1}^{s}\left[(t-1) b_{i i} t\right]\left[(t-1) b_{i i}+1\right]=t^{s} \bmod 2 .
$$

The sets $S_{0,1}$ and $S_{1,0}$ are empty. The sum $\sum_{s_{1,1}}$ is

$$
\sum_{i, k}\left\{\prod_{j=1 ; j \neq i}^{s}\left((t-1) b_{j j}-t\right)\right\}\left\{\prod_{j=1 ; j \neq k}^{s}\left((t-1) b_{j j}+1\right)\right\}(t-1)^{2} a_{i k} c_{k i}
$$

which $\bmod \left\{2,(t-1)^{4}\right\}$ is $t^{s-1}\left(1+t^{2}\right) c(V)$. Further similar calculation shows that $\sum_{s_{2,1}}+\sum_{s_{1,2}}=0$. (Essential use is made of the fact that $A$ and $C$ are symmetric.)

Before stating the lemma on normal bundles, recall that an $n$-dimensional bundle, $\xi^{n}$, over an $n$-sphere determines an element $\left[\xi^{n}\right] \in \pi_{n}\left(B_{S_{S O}}\right)$-where $B_{\text {SOn }}$ is a classifying space for the group SOn. We denote by $T_{n}$ the tangent bundle of $S^{n}$.

LEMMA ON NORMAL BUNDLES. Let $S^{n} \subset S^{2 n+1}$ be a differentiable imbedding, $\nu$ a never vanishing normal field, $\bar{S}^{n} \subset S^{2 n+1}$ a disjointly imbedded sphere obtained by "pushing" $S^{n}$ along $\nu$ and finally $\eta$, the complementary normal bundle-i.e., $\eta(x)$ $=$ the vectors normal to $S^{n}$ at $x$ but perpendicular to $v(x)$. Then

$$
[\eta]=L\left(S^{n}, \bar{S}^{n}\right)\left[T_{n}\right] \in \pi_{n}\left(B_{\text {SOn }}\right) \text {. }
$$

Proof. There is no loss in generality (see [3]) in assuming that the imbedding is the usual one-to wit $S^{n} \subset S^{n} \times R \subset R^{n+1}=R^{n+1} \times 0 \subset R^{n+1} \times R^{n}=S^{2 n+1}-\infty$. Thus we may refer the normal vector field, $v$, to the standard framing of this normal bundle - thus $v$ and $\eta$ are described completely by a function $f_{v}: S^{n} \rightarrow R^{n+1}-0$. Since the entities involved in our assertion are unchanged if we vary $v$ (through never-zero normal fields) we may assume that $f_{v}$ is a differentiable map to $S^{n}$ having the south pole as a regular value. Then it is clear that $\bar{S}^{n}$ intersects $D^{n+1} \times 0$ 
transversally once for each inverse image of the south pole and in fact $L\left(S^{n}, \bar{S}^{n}\right)$ $=$ the (algebraic) number of such inverse images $=$ the degree of $f_{v}$. On the other hand, we clearly now have $\eta=\left\{(x, v) \in S^{n} \times R^{n+1} \mid f_{v}(x) \perp v\right\}$ and this is obviously the "pull-back" under $f_{v}$ of the tangent bundle of $S^{n}$. Thus $[\eta]=\operatorname{deg} f_{v}\left[T_{n}\right]$.

\section{BIBLIOGRAPHY}

1. W. Browder, Homotopy type of differentiable manifolds, pp. 42-46, Colloq. Algebraic Topology, Aarhus University, 1962.

2. E. H. Brown, Jr. and F. P. Peterson, The Kervaire invariant of $(8 k+2)$-manifolds, Bull. Amer. Math. Soc. 71 (1965), 190-193.

3. A. Haeflinger, Plongements différentiables de variétés dans variétés, Comment Math. Helv. 36 (1961), 47-82.

4. M. A. Kervaire, Les noeuds de dimensions supérieures, Bull. Soc. Math. France 93 (1965), 225-271.

5. - The Browder-Novikov theorem, Lecture Notes, Tata Institute of Fundamental Research, Bombay, 1967.

6. M. A. Kervaire and J. Milnor, Groups of homotopy spheres, Ann. of Math. 77 (1963), 504-537.

7. J. Levine, Unknotting spheres in codimension two, Topology 4 (1965), 9-16.

8. J. Milnor, Differentiable structures on spheres, Amer. J. Math. 81 (1959), 962-972.

9. S. P. Novikov, Diffeomorphisms of simply connected manifolds, Soviet Math. Dokl. 3 (1962), 540-543.

10. R. Robertello, An invariant of knot cobordism, Comm. Pure Appl. Math. 18 (1965), 543-555.

NeW YoRK UNIVERSITY,

NeW YoRK, NeW YoRK

BRANDEIS UNIVERSITY,

Waltham, MassachusetTS 